EXTENDED REPORT

\title{
Changes of cartilage and bone markers after intra- articular glucocorticoid treatment with and without postinjection rest in patients with rheumatoid arthritis
}

\author{
T Weitoft, A Larsson, T Saxne, L Rönnblom
}

See end of article for authors' affiliations

Correspondence to: Dr Tomas Weitoft, Section of Rheumatology, Department of Internal Medicine, Gävle Hospital, 80187 Gävle, Sweden; tomas.weitoft@lg.se

Accepted 13 April 2005

Published Online First 20 April 2005

Background: Joint immobilisation improves the therapeutic effect of intra-articular glucocorticoid injection for knee synovitis. This may be due to retarded steroid resorption by immobilisation, a procedure that also could influence cartilage and bone metabolism.

Objective: To evaluate changes in cartilage and bone turnover after intra-articular glucocorticoid treatment for knee synovitis with and without postinjection rest.

Methods: 20 patients with rheumatoid arthritis and knee synovitis were randomised to 24 hour bed rest or to normal activity after intra-articular glucocorticoid treatment. Serum and urine markers of cartilage and bone turnover were studied for two weeks. Cartilage oligomeric matrix protein (COMP) was used as a marker of cartilage turnover, osteocalcin as marker of bone formation, and deoxipyridinoline (DPD) as marker of bone resorption.

Results: After the glucocorticoid injection COMP levels decreased in both groups $(p<0.001)$, but significantly more in resting patients. Serum osteocalcin levels decreased significantly $(p<0.001)$ without any difference between the groups. DPD was unchanged in both groups.

Conclusions: Intra-articular glucocorticoid treatment for knee synovitis reduced serum COMP, which suggests that such treatment may have a cartilage protective effect. The slightly larger decrease of serum COMP in the resting group may reflect a lower clearance of COMP from the joint cavity. Serum osteocalcin was temporarily reduced, indicating a reversible suppression of bone formation.

l ntra-articular glucocorticoid injection is an established treatment for synovitis in rheumatoid arthritis. Although 1 the purpose of such therapy is to treat the joint selectively, drainage of the injected substance from the joint has been shown. Thus, in studies where radioactive yttrium or gold was injected into knees of mobile patients, a significant leakage of these substances into inguinal lymph nodes and liver was found. ${ }^{12}$ Such leakage was not demonstrated in resting patients, and so a 24 to 48 hour postinjection rest after intra-articular therapy has been recommended. ${ }^{3}$ This immobilisation is supposed to retard drug resorption from the joint and increase the local therapeutic effect, resulting in better treatment outcome. This hypothesis is supported by investigations showing improved efficacy of intra-articular glucocorticoid injection treatment for knee synovitis if the injection is followed by a period of bed rest, compared with the response in mobilised patients. ${ }^{3}$

Several studies in healthy rabbits have shown that intraarticular glucocorticoids increase cartilage breakdown. ${ }^{5}$ The combination of exercise and repeated hydrocortisone injections leads to even more extensive cartilage damage. ${ }^{6}$ However, investigations in other species such as dogs and primates have not demonstrated any deleterious effect on the cartilage following repeated intra-articular glucocorticoid injections. ${ }^{7}$ In fact, such treatment may even protect from experimental osteoarthritis. ${ }^{8}$ In support of a chondroprotective effect by glucocorticoid treatment, low dose oral glucocorticoids, when added to methotrexate treatment, seem to prevent progression of joint erosions in patients with early rheumatoid arthritis. ${ }^{9}$ Furthermore, synovial fluid levels of core protein fragments of proteoglycan, a marker of cartilage turnover, decrease in synovial fluid after intraarticular glucocorticoid injection therapy for knee synovitis in patients with arthritis, suggesting a reduction in cartilage breakdown. ${ }^{10}$ Thus the effect on the joint tissues of intraarticular glucocorticoid injections may differ between species, but may also differ between joints with ongoing inflammation-that is, the joints that are injected in clinical practiceand normal joints injected in experimental settings. However, the question whether intra-articular steroid treatment can actually prevent or retard cartilage destruction in rheumatoid arthritis remains unresolved.

The development of assays for quantification of tissue markers for cartilage and bone in serum has facilitated the monitoring of tissue turnover in the joint and also has shed light on the transport of molecules, or their fragments, from the joint into the circulation. ${ }^{11}{ }^{12}$ In the present study we used a set of such markers to assess the dynamics of cartilage and bone turnover in relation to an intra-articular glucocorticoid injection. We examined the impact on both cartilage and bone turnover by comparing patients subjected to mobilisation or immobilisation after the injection. Cartilage oligomeric matrix protein (COMP) was used to evaluate cartilage turnover..$^{13}$ The bone formation marker serum osteocalcin and the bone resorption marker urinary deoxypyridinoline corrected for creatinine (DPD) were used to assess bone turnover. ${ }^{12}$

\section{METHODS}

Patients and clinical and radiographic assessments Patients fulfilling the 1987 American College of rheumatology (ACR) criteria for rheumatoid arthritis, ${ }^{14}$ and with signs and symptoms of knee synovitis requiring treatment with

Abbreviations: COMP, cartilage oligomeric matrix protein; DPD, deoxipyridinoline 
Table 1 Patient characteristics

\begin{tabular}{|c|c|c|c|}
\hline & Rest group $(n=10)$ & Mobile group ( $\mathrm{n}=$ & \\
\hline $\begin{array}{l}\text { Age (years)* } \\
\text { Disease duration (years) } \\
\text { Serum C reactive protein }(\mathrm{mg} / \mathrm{l})^{*} \\
\text { DAS28 } \\
\mathrm{HAQ}^{*} \\
\text { Volume of effusion }(\mathrm{ml})^{*} \\
\text { Larsen-Dale score }{ }^{*}\end{array}$ & $\begin{array}{l}62(37 \text { to } 75) \\
10(0 \text { to } 53) \\
18(5 \text { to } 48) \\
4.88(2.79 \text { to } 5.76) \\
1.2(0.88 \text { to } 1.63) \\
18.5(0 \text { to } 36) \\
1.5(0 \text { to } 4)\end{array}$ & $\begin{array}{l}59(30 \text { to } 76) \\
14.5(2 \text { to } 48) \\
46.5(5 \text { to } 158) \\
4.99(3.3 \text { to } 6.4) \\
1.2(0.5 \text { to } 2.13) \\
13.5(3 \text { to } 48) \\
1.5(0 \text { to } 5)\end{array}$ & $\begin{array}{l}\text { NS } \\
\text { NS } \\
\text { NS } \\
\text { NS } \\
\text { NS } \\
\text { NS } \\
\text { NS }\end{array}$ \\
\hline \multicolumn{4}{|c|}{$\begin{array}{l}\text { Values are median (range). } \\
\text { *Student's } t \text { test used for comparison. } \\
\text { †Mann-Whitney U test used for comparison. } \\
\text { DAS28, } 28 \text { joint disease activity score; HAQ, Health Assessment Questionnaire. }\end{array}$} \\
\hline
\end{tabular}

intra-articular glucocorticoids at the outpatient rheumatology departments in Gävle and Uppsala, were asked to participate in the study. Patients treated with tumour necrosis factor blockers, systemic glucocorticoids during the past year, an intra-articular glucocorticoid injection in the knee during the past six months, or those with any intra-articular injection the past two months were excluded, as were patients in functional class 4 according to Steinbrocker. ${ }^{15}$

Twenty consecutive patients were included and randomly allocated to 24 hour postinjection bed rest or normal activity. To avoid joint movement and to assure that no weight bearing occurred, patients allocated to bed rest were supervised in hospital.

The injection procedure was undertaken with the patient in a supine position. The knee joint was entered by a lateral approach, using a $0.7 \times 40 \mathrm{~mm}$ needle. When the joint had been aspirated of as much synovial fluid as possible, $20 \mathrm{mg}$ of triamcinolone hexacetonide (Lederspan ${ }^{\circledR}$ ) were injected. Non-fasting serum samples were collected immediately before the injection and after 24 hours, 48 hours, one week, and two weeks. The injections were given and samples collected at 08.00 hours on all occasions. At the same time the first morning void of urine was collected.

Three patients, two in the rest group, were at one occasion each unable to give a morning urine sample. The COMP samples from one patient in the mobile group were destroyed in a transportation failure.

To evaluate the level of disability at baseline, the Swedish version of the Health Assessment Questionnaire (HAQ) was used..$^{16}$ The number of tender and swollen joints was counted, and the disease activity score (DAS28) ${ }^{17}$ was calculated. All patients underwent a radiological examination of the treated knee. The radiographs were graded by an independent radiologist according to Larsen-Dale. ${ }^{18}$

The study protocol was approved by the committee of ethics, Faculty of Medicine, Uppsala University, and informed consent was obtained from the patients.

\section{Biochemical analyses}

The serum and synovial fluid samples were centrifuged for 10 minutes at $1800 \times g$ within one hour and stored at $-70^{\circ} \mathrm{C}$ until all samples had been collected and laboratory processing could be undertaken. Erythrocyte sedimentation rate (ESR) and serum $C$ reactive protein were assayed by standard methods. COMP was quantified by sandwich enzyme linked immunosorbent assay (ELISA) (Anamar Medical, Lund, Sweden), osteocalcin by immunoassay (Elecsys ${ }^{\circledR}$, Roche Diagnostics, Mannheim, Germany), and DPD by ELISA (Metra DPD, Quidel Corporation, San Diego, California, USA). The total analytical imprecision (coefficient of variation, CV) for the cartilage and bone turnover markers was $<5 \%$ for COMP, $<2 \%$ for osteocalcin, and $<8 \%$ for DPD. All laboratory testing was done blind, without knowledge of the clinical data or study group.
Limits for normal range were provided by the manufacturers, except for DPD which was defined in 77 healthy Swedish individuals with normal bone density in Uppsala (Larsson A, Brahm H, Ljunggren Ö, 1998: unpublished data). The mean (SD) serum COMP level in 336 Swedish blood donors was 10.2 (2.9) U/l (data provided by Anamar Medical). The normal range in women for osteocalcin was 15-46 $\mu \mathrm{g} / \mathrm{l}$ and for DPD 5.4 (3.0) nmol/mmol creatinine.

\section{Statistical calculations}

For comparison of baseline characteristics between the groups, Student's $t$ test and the Mann-Whitney $U$ test were used when appropriate. To test for significant differences of the changes of levels of COMP, osteocalcin, and DPD between the groups, analysis of covariance with baseline as covariate was used. All tests were two tailed. Comparisons within a group were carried out using a one sample $t$ test. A probability $(p)$ value of $<0.05$ was considered significant. The statistical analyses were made by the statistical computer software program SPSS, version 11.5 for Windows NT (SPSS Inc, Chicago, Illinois, USA).

\section{RESULTS}

\section{Patients}

Patient characteristics are shown in table 1. No significant differences were observed between the patient groups. The participants were all female except for one man in the mobile group. Eight patients in the resting group and seven in the mobile group were treated with disease modifying antirheumatic drugs. Only four patients, of whom three were in the mobile group, had ever previously been exposed to systemic glucocorticoids, and only two patients in each group had

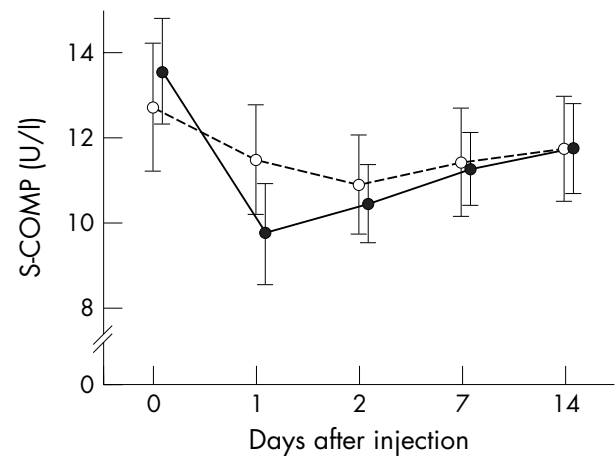

Figure 1 Mean serum cartilage oligomeric matrix protein (S-COMP) levels for mobile patients (empty circles) and patients with 24 hour postinjection rest (filled circles) during a two week period after intraarticular glucocorticoid treatment for knee synovitis. The results are means, error bars $=\mathrm{SEM}$, for 10 samples in the resting group and nine in the mobile group. 
been treated with intra-articular glucocorticoids in another joint during the past six months.

\section{Cartilage turnover}

Before injection, the COMP levels in synovial fluid (mean (SD)) did not differ between the two patient groups: 56.6 (24.0) $\mathrm{U} / \mathrm{l}$ in the resting group and $58.4(22.8) \mathrm{U} / \mathrm{l}$ in the mobile group. These concentrations are within the range typically seen in rheumatoid synovial fluids using the original inhibition ELISA ${ }^{13}$ and the assay used in the present study (Saxne T, unpublished data).

The serum concentrations of COMP at baseline were within the normal range for both resting and mobile patients, at 13.5 (3.9) $\mathrm{U} / \mathrm{l}$ and 12.7 (4.5) U/l, respectively. After injection, serum COMP decreased within 24 hours $(\mathrm{p}<0.001)$ in both patient groups (fig 1). The decrease was significantly more pronounced in the resting group $(p=0.007)$. The results for each patient are shown in fig 2 . In the mobile patients the minimum COMP level was found at 48 hours after the injection, and after one week serum COMP was no longer decreased compared with baseline. In the resting patients, on the other hand, significantly decreased levels were noted throughout the study.

\section{Bone formation}

The mean serum osteocalcin was within normal range in both patient groups before the glucocorticoid injection (30.6 (7.6) and 25.1 (5.6) $\mu \mathrm{g} / \mathrm{l}$ respectively, NS). After injection the levels decreased within 24 hours in both groups $(p<0.001)$, and there was no difference between resting and mobile patients (fig 3). In mobile individuals significantly reduced levels could be seen for 48 hours and in resting patients during the first week.

\section{Bone resorption}

Before injection, the mean urine concentrations of DPD were above the normal range in both groups (10.4 (4.9) and 10.3 (4.7) $\mathrm{nmol} / \mathrm{mmol}$ creatinine), but no difference between mobile and resting patients could be detected. There was no significant change in the levels in either group during the observation period.

\section{DISCUSSION}

One of the principal findings in this study was that a single intra-articular glucocorticoid injection induced a significant reduction in serum COMP levels within 24 hours in rheumatoid patients with knee synovitis. Furthermore, the

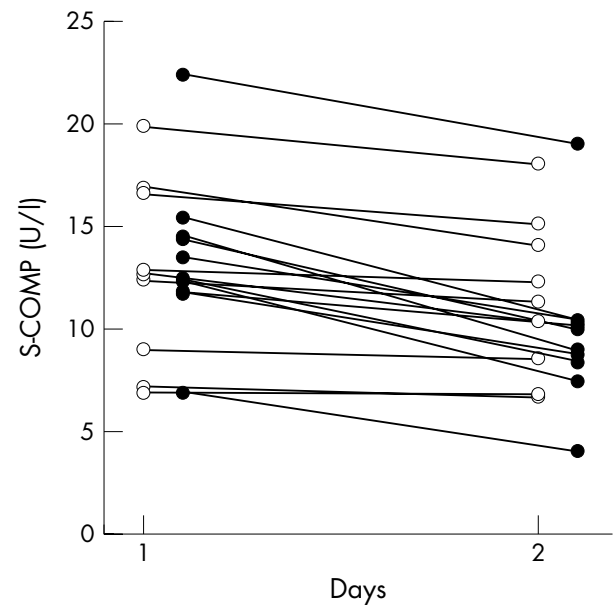

Figure 2 The change of serum cartilage oligomeric matrix protein (SCOMP) levels for each patient during the first 24 hours.

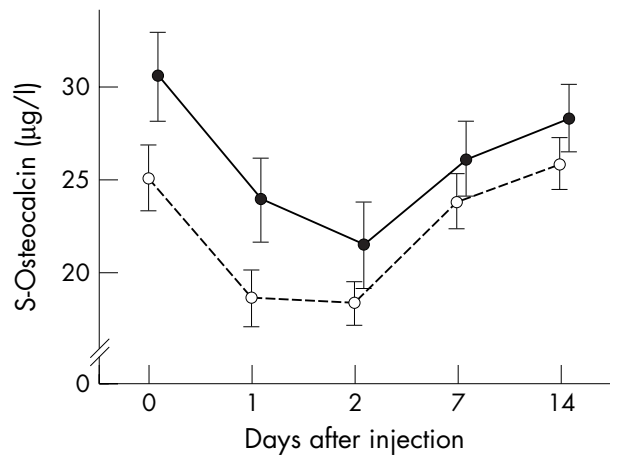

Figure 3 Mean serum osteocalcin (S-osteocalcin) levels for mobile patients (empty circles) and patients with 24-hour postinjection rest (filled circles) during a two week period after intra-articular glucocorticoid treatment for knee synovitis. The results are means, error bars = SEM, for 10 samples in each group.

reduction persisted during the study period of two weeks, and the reduction in serum levels was more pronounced in the resting patients. In previous studies in experimental arthritis it has been shown convincingly that changes in serum COMP parallel histological changes in cartilage morphology. ${ }^{19-21}$ Furthermore, cytokine modulating treatment which reduces cartilage pathology also reduces serum COMP, whereas treatment which only reduces inflammation without influencing cartilage pathology does not influence serum COMP. ${ }^{20}$ Also, in type II collagen induced arthritis in rats, the retardation of cartilage damage induced by glucocorticoid treatment is accompanied by lower serum COMP in the treated animals. ${ }^{21}$ In studies on rheumatoid patients, both low dose prednisolone ( $7.5 \mathrm{mg}$ daily orally) and intramuscular depot methyl prednisolone acetate $120 \mathrm{mg}$ reduced serum COMP (Saxne T, Chikanza IC, unpublished data). In further support of the potential usefulness of serum COMP in evaluating treatment, it was recently shown that anti-tumour necrosis factor therapy in rheumatoid patients reduced serum COMP regardless of the clinical response to the treatment. ${ }^{22}$

Although the results for COMP in this study are in line with previous observations in other experimental and clinical settings, the results should be interpreted with caution. It seems unlikely that the initial fairly dramatic fall in serum levels results from a direct effect on the cartilage. It may well be a reflection of reduced synovial permeability and the efflux of fragments out of the synovial cavity induced by a rapid anti-inflammatory effect of the glucocorticoid injection. A role for loading related factors in the initial phase after glucocorticoid injection is also suggested by the difference in COMP levels between resting and mobile patients. The local anti-inflammatory effect may be more pronounced in the resting group of patients, assuming that the glucocorticoid is retained in the joint for a longer period.

As serum COMP levels remained low and did not differ between the groups by day 14 , it is possible that by this stage there is a positive effect on cartilage turnover resulting in a lower release of COMP molecules from the cartilage. This would fit with the results obtained in experimental arthritis models and in unpublished clinical studies and suggests a chondroprotective potential of the treatment.

The intra-articular glucocorticoid treatment markedly suppressed the osteocalcin levels in both patient groups, but no difference between mobile and resting individuals was observed. This suggests a temporary reduction in bone formation by intra-articular glucocorticoid treatment, and our results support previous investigations demonstrating a clear impact on serum osteocalcin levels by glucocorticoids. ${ }^{23}$ 
In patients with early rheumatoid arthritis, for example, oral prednisolone treatment at a dose of $7.5 \mathrm{mg}$ daily reduced the osteocalcin levels by $25 \%,{ }^{24}$ a decrease of the same magnitude as in our study. The bed rest procedure did not influence the osteocalcin levels, indicating that the assumed difference in steroid resorption is not large enough to influence bone formation.

Bone formation is a slow process, and rapid changes in osteocalcin may not reflect long term osteoblast activity. ${ }^{25}$ The observed temporary decrease of osteocalcin may therefore have little or no impact on the risk of the patient developing osteoporosis.

The urinary secretion of DPD was unchanged in both patient groups during the whole study, in agreement with previous results. ${ }^{24}{ }^{26}$ Bed rest for 24 hours did not influence the levels. The findings in our study thus support the view that intra-articular glucocorticoid treatment mainly affects bone formation and not bone resorption.

In a recent study on rheumatoid patients it was reported that intravenous glucocorticoid pulse therapy preserved bone mineral density, in contrast to the controls who used continuous oral treatment. ${ }^{27}$ There are obvious similarities between intermittent glucocorticoid pulse therapy and intraarticular treatment. The short term influence on bone metabolism after intra-articular administration may suggest that injection treatment reduces the risk of steroid induced osteoporosis. However, further studies are needed to clarify this.

\section{Conclusions}

In summary, our results suggest that intra-articular glucocorticoid treatment of knee synovitis may reduce cartilage breakdown. Furthermore, if immobilisation of the patient for a period of 24 hours is included in the injection protocol, the reduction in cartilage breakdown may be even more pronounced. Bone formation is temporarily inhibited by the glucocorticoid injection, and bone resorption is unaffected, independent of the immobilisation procedure.

The metabolic effects on cartilage and bone metabolism after intra-articular glucocorticoid treatment seem to be short term and reversible. Our interpretation of the present results is that intra-articular glucocorticoid therapy in rheumatoid arthritis is not only an effective symptomatic treatment but may also be cartilage protective.

\section{ACKNOWLEDGEMENTS}

We wish to thank Britt-Inger Nilsson, Åsa Olsson, Gunilla Andersson, and Helena Sjösten for helping us with collecting of blood and urine samples. We are grateful to Dr Modolv Saebö for examining the radiographs. Hans Högberg, FOU-forum Gävleborg, has been of great help for statistical analysis.

The study was supported by grants from the Swedish Research Council, FOU-forum Gävleborg, the Swedish Rheumatism Foundation, the 80-Year Foundation of King Gustav V and WyethLederle.

\section{Authors' affiliations}

T Weitoft, Section of Rheumatology, Department of Internal Medicine, Gävle Hospital, Gävle, Sweden

A Larsson, Department of Medical Science, Clinical Chemistry, Uppsala University Hospital, Uppsala, Sweden

T Saxne, Department of Rheumatology, Lund University Hospital, Lund, Sweden

L Rönnblom, Department of Medical Science, Section of Rheumatology, Uppsala University Hospital

\section{REFERENCES}

1 Topp JR, Cross EG, Fam AG. Treatment of persistent knee effusions with intraarticular radioactive gold. Can Med Assoc J 1975;112:1085-9.

2 Oka M. Radiation synovectomy of the rheumatoid knee with yttrium 90. Ann Clin Res 1975;7:205.

3 Chakravarty K, Pharoah PD, Scott DG. A randomized controlled study of postinjection rest following intra-articular steroid therapy for knee synovitis. Br J Rheumatol 1994;33:464-8.

4 Neustadt DH. Synovitis of the knee. Effects of postinjection rest. Clin Rheumatol Pract 1985;3:65-8.

5 Behrens F, Shepard N, Mitcell N. Alteration of rabbit articular cartilage of intra-articular injections of glucocorticoids. J Bone Surg 1975;57A:70-6.

6 Gogia P, Brown M, Al-Obaidi S. Hydrocortisone and exercise effects on articular cartilage in rats. Arch Phys Med Rehabil 1993;74:463-6.

7 Gibson T, Burry HC, Poswillo D, Glass J. Effect of intra-articular corticosteroid injections on primate cartilage. Ann Rheum Dis 1976;36:74-9.

8 Pelletier J-P, Martel-Pelletier J. Protective effects of corticosteroids on cartilage lesions and osteophyte formation in the Pond-Nuki dog model of osteoarthritis. Arthritis Rheum 1989;32:181-93.

9 Kirwan JR. The effect of glucocorticoids on joint destruction in rheumatoid arthritis. The Arthritis and Rheumatism Council Low-Dose Glucocorticoid Study Group. N Engl J Med 1995;20:142-6.

10 Saxne T, Heinegard D, Wollheim F. Therapeutic effects on cartilage metabolism in arthritis as measured by release of proteoglycan structures into synovial fluid. Ann Rheum Dis 1986;45:491-7.

11 Saxne T, Mansson B. Molecular markers for assessment of cartilage damage in rheumatoid arthritis. In: Firestein GS, Panayi GS, Wollheim FA, eds. Rheumatoid arthritis: new frontiers in pathogenesis and treatment. Oxford: Oxford University Press, 2000:291-304.

12 Garnero P, Rousseau JC, Delmas PD. Molecular basis and clinical use of biochemical markers of bone, cartilage, and synovium in joint diseases. Arthritis Rheum 2000;43:953-68

13 Saxne T, Heinegard D. Cartilage oligomeric protein a novel marker of cartilage turnover detectable in synovial fluid and blood. Br J Rheumatol 1992;31:583-91.

14 Arnett FC, Edworthy SM, Bloch DA, Shane DA, Fries JF, Cooper NS, et al. The American Rheumatism Association 1987 revised criteria for the classification of rheumatoid arthritis. Arthritis Rheum 1988;31:315-24.

15 Steinbrocker O, Traeger $\mathrm{CH}$, Betterman RC. Therapeutic criteria in rheumatoid arthritis. JAMA 1949;140:659-62.

16 Ekdahl C, Eberhardt K, Andersson SI, Svensson B. Assessing disability in patients with rheumatoid arthritis. Scand J Rheumatol 1988;17:263-71.

17 Prevoo ML, van't Hof MA, Kuper HH, van Leeuwen MA, van de Putte LB, van Riel PL. Modified disease activity scores that include twenty-eight joint counts. Development of and validation in a prospective longitudinal study of patients with rheumatoid arthritis. Arthritis Rheum 1995;38:44-8.

18 Larsen A, Dale K. Standardized radiological examination of rheumatoid arthritis in therapeutical trials. In: Dumonde DC, Jasani MK, eds. The recognition of antirheumatic drugs. Lancaster: MTP Press, 1978:285-92.

19 Vingsbo-Lundberg C, Saxne T. Increased serum levels of COMP in chronic erosive arthritis in rats. Arthritis Rheum 1998;41:544-50.

20 Joosten L, Helse MM, Saxne T, van der Loo, Heinegard D, van den Bergh W. IL-1 $\alpha \beta$ blockade prevents cartilage and bone destruction in murine type II collagen-induced arthritis, whereas TNF- $\alpha$ blockade only ameliorates joint inflammation. J Immunol 1999; 163:5049-55.

21 Larsson E, Erlandsson-Harris H, Klareskog L, Larsson A, Mansson B, Saxne T. Corticosteroid treatment of experimental arthritis retards cartilage destruction as determined by histology and serum COMP. Rheumatology 2004; 180:62-5.

22 Crnkic M, Mansson B, Larsson L, Geborek P, Heinegard D, Saxne T. Serum cartilage oligomeric matrix protein (COMP) decreases in rheumatoid arthritis patients treated with infliximab or etanercept. Arthritis Res Ther 2003;5:181-5.

23 Emkey RD, Lindsay R, Lyssy J, Weisberg JS, Dempster DW, Shen V. Systemic effect of intra-articular administration of corticosteroid on markers of bone formation and bone resorption in patients with rheumatoid arthritis. Arthritis Rheum 1996:39:277-82.

24 Sharif M, Salisbury C, Taylor DJ, Kirwan JR. Changes in biochemical markers of joint tissue metabolism in a randomized controlled trial of glucocorticoid in early rheumatoid arthritis. Arthritis Rheum 1998;41:1203-9.

25 Heart S, Eastell R. Biochemical markers of bone turnover. Curr Opin Nephrol Hypertens 1999;8:421-7.

26 Lems WF, Gerritz MI, Jacobs JWG, van Vugt RM, van Rijn HJ, Bijlsma JW. Changes in (markers of) bone metabolism during high dose corticosteroid pulse treatment in patients with rheumatoid arthritis. Ann Rheum Dis 1996;55:288-93.

27 Frediani B, Falsetti P, Bisogno S Baldi F, Acciai C, Filippou G, et al. Effects of high dose methylprednisolone pulse therapy on bone mass and biochemical markers of bone metabolism in patients with active rheumatoid arthritis: a 12-month randomised and controlled study. J Rheumatol 2004;31:1083-7. 